\title{
Evidence for proteasome dysfunction in cytotoxicity mediated by anti-Ras intracellular antibodies
}

\author{
Alessio Cardinale, Ilaria Filesi, Sonia Mattei and Silvia Biocca
}

Department of Neuroscience, University of Rome 'Tor Vergata', Rome, Italy

\begin{abstract}
Anti-Ras intracellular antibodies inhibit cell proliferation in vivo by sequestering the antigen and diverting it from its physiological location [Lener, M., Horn, I. R., Cardinale, A., Messina, S., Nielsen, U.B., Rybak, S.M., Hoogenboom, H.R., Cattaneo, A., Biocca, S. (2000) Eur. J. Biochem. 267, 1196-1205]. Here we demonstrate that strongly aggregating single-chain antibody fragments $(\mathrm{scFv})$, binding to Ras, induce apoptosis, and this effect is strictly related to the antibody-mediated aggregation of p21Ras. Proteasomes are quickly recruited to the newly formed aggregates, and their activity is strongly inhibited. This leads to the formation of aggresome-like structures, which become evident in the vast majority of apoptotic cells. A combination of anti-Ras scFv
\end{abstract}

fragments with a nontoxic concentration of the proteasome inhibitor, lactacystin, markedly increases proteasome dysfunction and apoptosis. The dominant-negative H-ras (N17-H-ras), which is mostly soluble and does not induce aggresome formation or inhibit proteasome activity, only affects cell viability slightly. Together, these observations suggest a mechanism linking antibody-mediated Ras aggregation, impairment of the ubiquitin-proteasome system, and cytotoxicity.

Keywords: aggresome; anti-p21Ras; apoptosis; proteasome; scFv fragment.
Ras is a membrane-bound GTP/GDP-binding protein which functions as a molecular switch in a large network of signaling pathways [1]. Mutations in the ras gene have been identified in about $30 \%$ of all human cancers, indicating that this molecule is a preferential target for the development of anticancer strategies. Indeed, Ras protein has been inhibited through different approaches such as ribozymes, antisense oligonucleotides, farnesyl-transferase inhibitors, dominant-negative mutants, and intracellular antibodies [2,3]. Results obtained to date indicate that inhibition of Ras activity suppresses cell proliferation and induces regression in a broad range of tumors.

Intracellular antibodies, in particular single-chain $\mathrm{Fv}$ (scFv) fragments, have been successfully expressed inside cells to ablate the function of several antigens in different subcellular compartments [4,5], including p21Ras. The efficacy of blocking Ras by the neutralizing anti-Ras Y13259 has been documented both in tumor cell lines and animal models [6,7]. Moreover, we have demonstrated that phage-derived anti-Ras scFv fragments, with high variability in terms of solubility and intracellular stability, can sequester the antigen in intracellular aggregates, divert it from its physiological location, and inhibit its function.

Correspondence to S. Biocca, Department of Neuroscience, University of Rome 'Tor Vergata', Via Montpellier 1, 00133 Roma, Italy. Fax: + 3967259 6407, Tel.: + 39672596428 ,

E-mail: biocca@med.uniroma2.it

Abbreviations: scFv, single-chain variable fragment; ECL, enhanced chemiluminescence; UPS, ubiquitin-proteasome system; HP1 $\beta$, heterochromatin protein $1 \beta ; \beta \mathrm{Gal}, \beta$-galactosidase; NGF, nerve growth factor; GFP, green fluorescent protein; scPs, single-chain proteasome substrate.

(Received 28 April 2003, accepted 18 June 2003)
Antigen-specific coaggregation of several nonneutralizing $\mathrm{scFv}$ fragments with the corresponding protein led to prospect the antibody-directed aggregation of the antigen as a general mode of action of intracellular antibodies that could be exploited for intracellular antibody-based phenotypic knock-outs [6,8-11].

A common feature of the phenomenon of aggregation is the formation of pericentriolar membrane-free, cytoplasmic inclusions, named aggresomes. Consistent with their formation as a part of a response to cellular stress, aggresomes are enriched in proteasome subunits, ubiquitin and molecular chaperones $[12,13]$. These structures are considered symptoms of the impairment of the ubiquitin-proteasome system (UPS). This is a nonlysosomal protein degradation machine by which many critical regulatory proteins involved in the regulation of cell proliferation and survival are degraded [14,15]. Indeed, proteasome inhibitors block cell proliferation and induce apoptosis in cancer cells, providing a novel class of potent antitumor agents [16,17].

The fact that scFvs are ubiquitinated and tend to aggregate suggests that these molecules are prone to misfold and represent specific substrates of the UPS. In fact, targeted inhibition of the $26 \mathrm{~S}$ proteasome increases the formation of large perinuclear scFv aggresomes and induces the accumulation of multi-ubiquitinated $\mathrm{scFv}$ fragments [18].

In this paper we report that the aggregating anti-Ras $\mathrm{scFV}$ fragments induce apoptosis in a high percentage of transfected cells and inhibit cell growth in different cell lines. This phenomenon is accompanied by the formation of aggresomes and recruitment of proteasomes to the newly formed aggregates. Proteasome activity is strongly inhibited, as demonstrated by the accumulation of an exogenous proteasome substrate in an in vivo proteasome activity assay. Furthermore, combined treatment of a nontoxic 
concentration of lactacystin with aggregating anti-Ras scFvs induces a synergistic effect on apoptosis. Finally, the dominant-negative H-ras (N17-H-ras), notwithstanding its ability to block Ras function in vivo [19], only slightly affects cell viability and proteasome activity. These observations suggest that antibody-mediated Ras sequestration induces an apoptotic phenotype strictly related to the impairment of proteasome activity.

\section{Materials and methods}

\section{DNA constructs}

Anti-(Ras 1), anti-(Ras 5), anti-(Ras 6), anti-[nerve growth factor $(\mathrm{NGF})]$, anti-[ $\beta$-galactosidase $(\beta \mathrm{Gal})] \mathrm{scFv}$ fragments and the single-chain proteasome substrate (scPs) $\alpha \mathrm{D} 11-\mathrm{sec}$ were cloned as previously described $[6,9,20]$. To generate the anti- $\beta$ Gal-green fluorescent protein (GFP), anti(Ras 1)-GFP and anti-(Ras 5)-GFP constructs bearing cytomegalovirus promoter, the pscFvexp-cyt-163R4-GFP (anti- $\beta$ Gal), pscFvexp-anti-Ras1-GFP and pscFvexp-antiRas5-GFP [18] were PCR-amplified into the $B g / \mathrm{II}-\mathrm{XbaI}$ sites of the pEGFPN1 vector (Clontech). The following oligonucleotides were designed: 5'-GGAAGATCTCAC GTGGCCACCATG and 3'-GCTCTAGATTACTTGTA CAGCTCGTCCAT.

The dominant-negative H-ras (N17) fused to GFP was generated by subcloning the HindIII-BamHI fragment derived from pXCR Asn17 [19], containing the N17-Hras mutant, into the $\mathrm{pEGFPC1}(\mathrm{Clontech})$ vector.

\section{Cell lines, transfection and drug treatment}

NIH 3T3 Ki-Ras fibroblasts (kindly provided by C. Schneider, CIB, Trieste, Italy), human tumor pancreatic carcinoma Ger and MIA PaCa-2 (kindly provided by R. Orlandi, INT, Milan Italy) and human tumor breast adenocarcinoma cell lines MDA-MB-231 (kindly provided by F. Cozzolino, Dept. Exp. Med., University of Rome 'Tor Vergata', Italy) were grown in Dulbecco's modified Eagle's medium supplemented with $10 \%(\mathrm{v} / \mathrm{v})$ fetal bovine serum. NIH 3T3 Ki-Ras fibroblasts were transiently transfected with Superfect (Qiagen) as described [9], and Ger, MIA PaCa-2 and MDA-MB-231 cells were transfected with the Lipofectamine 2000 reagent (Life Technologies) following the manufacturer's instructions. Cells were analysed 1 and 2 days after transfection, as specified for each case. Lactacystin (Calbiochem) was used as specified in each experiment.

\section{Western blot analysis and immunoprecipitation}

Cells were harvested and analysed $48 \mathrm{~h}$ after transfection. Lysis, extraction of cellular proteins, immunoprecipitation and Western blotting have been described previously [18]. The following primary antibodies were used in this study: monoclonal mouse anti-myc IgG (9E10), rabbit polyclonal anti-GFP IgG (Clontech), monoclonal mouse anti-ubiquitin IgG (Calbiochem), monoclonal rat anti-[heterochromatin protein $1 \beta(\mathrm{HP} 1 \beta)$ ] IgG (MAC 353, kindly provided by $P$. Singh, Roslin Institute, Midlothian, UK) [21]. Horseradish peroxidase-conjugated goat anti-mouse, anti- rabbit IgG (Amersham Pharmacia Biotech) and anti-rat IgG (Pierce) were used as secondary antibodies. Immunoblots were visualized using the ECL detection kit (Amersham Pharmacia Biotech).

\section{Immunofluorescence microscopy}

Cells were grown on glass coverslips coated with poly(Llysine), then fixed and permeabilized as described [9]. Incubation with high affinity-purified mouse anti-myc IgG $9 \mathrm{E} 10$ and rabbit polyclonal antibody to $20 \mathrm{~S}$ proteasome $\alpha / \beta$ subunits (Affiniti Research Products, Golden, CO, USA) was carried out at room temperature for $1 \mathrm{~h}$; Texas-Red goat anti-mouse IgG (Calbiochem) and Cy2-conjugated donkey anti-rabbit IgG (Jackson ImmunoResearch) were used as secondary antibodies.

Double immunofluorescence was viewed with a DMRA Leica fluorescence microscope equipped with a DC250 CCD camera, using a $100 \times 1.3-0.6$ oil immersion objective. Images were recorded and analysed with Leica QFLUORO software.

\section{In situ identification of apoptotic cells}

Apoptotic cells were detected using annexin V-FLUOS, annexin V-Alexa 568 (Roche) and the blue fluorescent dye Hoechst 33342 (Sigma). Briefly, transfected cells were rinsed twice in $\mathrm{NaCl} / \mathrm{P}_{\mathrm{i}}$ and incubated with Hepes solution (Roche) containing annexin V and Hoechst 33342 for $15 \mathrm{~min}$. Then, cells were fixed with ice-cold acetone/ methanol solution $(7: 3, \mathrm{v} / \mathrm{v})$ at $-20{ }^{\circ} \mathrm{C}$ for $30 \mathrm{~min}$, airdried for $15 \mathrm{~min}$, washed three times in $\mathrm{NaCl} / \mathrm{P}_{\mathrm{i}}$ and incubated with anti-myc IgG (9E10) to visualize scFvexpressing cells. The results shown in Figs 1, 4 and 5 are the average from three different experiments. At least 150 positively transfected cells for each plasmid were counted.

\section{Colony-forming assay}

Subconfluent monolayer cultures were transfected with different $\mathrm{scFv}$ fragments. The next day, cultures were trypsinized to generate a single cell suspension, and $20 \times 10^{4}$ cells were seeded into three $100-\mathrm{mm}$ tissue culture dishes. After 15-20 days of G418 selection, cells were fixed and stained with a solution of $0.4 \%$ Coomassie blue and $50 \%$ 2-propanol for $5 \mathrm{~min}$. Only colonies with more than 50 cells were counted. Data represent the mean of two independent experiments.

\section{In vivo proteasome activity assay}

NIH 3T3 Ki-Ras fibroblasts $\left(1 \times 10^{6}\right)$ were cotransfected with scPs and different GFP-tagged $\mathrm{scFv}$ fragments at a $1: 0.5$ or $1: 1$ DNA ratio. At $48 \mathrm{~h}$ after transfection, cells were lysed in $4 \times$ sample buffer $(500 \mathrm{~mm}$ Tris $/ \mathrm{HCl}$, $\mathrm{pH} 6.8,4 \%$ SDS, $40 \mathrm{~mm}$ dithiothreitol and $20 \%$ glycerol) for $30 \mathrm{~min}$ on ice, centrifuged for $5 \mathrm{~min}(15000 \mathrm{~g})$ at room temperature, boiled for $8 \mathrm{~min}$, and analysed by SDS/PAGE. Western blotting was carried out by using the mouse monoclonal anti-myc IgG 9E10. One-twentieth of the original cell suspension was used for protein determination (Bradford reagent; Bio-Rad). 


\section{Results}

\section{Aggregating anti-Ras scFvs induce apoptosis and inhibit cell growth}

We noticed that cells expressing the aggregating anti-Ras intracellular antibodies did not thrive, with many of the cells exhibiting cell shrinkage. To study the cytotoxicity of these scFvs, we transfected mouse NIH $3 \mathrm{~T} 3 \mathrm{Ki}-\mathrm{Ras}$ fibroblasts with three previously characterized constructs [9]: (a) the strongly aggregating anti-(Ras 5), which does not neutralize Ras function in vitro; (b) the strongly aggregating anti(Ras 1), which is derived from the well-characterized neutralizing Y13-259 monoclonal antibody; (c) the mostly soluble and non-neutralizing anti-(Ras 6) scFv fragment.

We quantified the dying cells by using two markers of apoptosis: annexin V, which detects phosphatidylserine translocation from the inner side to the outer layer of plasma membrane and represents an early marker of apoptosis; blue-fluorescent Hoechst 33342 dye, which stains the condensed chromatin of apoptotic cells more brightly than the chromatin of nonapoptotic cells. On the basis of the combined staining patterns of these apoptotic markers, we were able to distinguish between normal and apoptotic cells. As seen in Fig. 1A, the percentage of apoptotic cells was higher when transfected with aggregating anti-Ras scFvs and varied between $30 \%$ and $33 \%$ with anti-(Ras 5) and $45 \%$ and $50 \%$ with anti-(Ras 1$)$. These numbers were calculated on the basis of annexin V/Hoechst-positive cells, $48 \mathrm{~h}$ after transfection. Only a few annexin V-positive cells were also positive for propidium iodide (data not shown), indicating that the cells examined were not necrotic but actually undergoing a process of programmed cell death. The process peaked at $16 \mathrm{~h}$ of transfection, when detected by annexin $\mathrm{V}$ and was not time dependent. In contrast, much lower levels of apoptosis were observed in nontransfected cells or cells transfected with the anti-(Ras 6) and the two irrelevant scFv constructs (anti- $\beta$ Gal and anti-NGF). Similar results were obtained by transfecting two human pancreatic tumor cell lines, Ger and MIA PaCa-2, suggesting that the apoptotic response mediated by aggregating anti-Ras scFvs is not cell specific but, rather, a general mechanism (Table 1). To confirm the link between the observed apoptotic phenotype and the process of anti-Ras scFv aggregation, NIH 3T3 Ki-Ras fibroblasts were transfected with plasmids coding for anti-(Ras 1), anti-(Ras 6) and anti-( $\beta \mathrm{Gal})$ scFvs and costained with anti-myc $9 \mathrm{E} 10$ IgG and the two markers of apoptosis, annexin $\mathrm{V}$ and Hoechst. As shown in Fig. 1B, cells expressing the aggregating anti-(Ras 1) molecules were round, exhibited aggregates (d) and were apoptotic (e and f). In the case of anti-(Ras 6), only the cell that exhibited scFv aggregates was apoptotic (see blank arrow in $\mathrm{g}, \mathrm{h}$ and $\mathrm{i}$ ). It is worth noting that the anti-( $\beta \mathrm{Gal})$-expressing cells and the vast majority of anti-(Ras 6)-expressing cells were healthy and showed diffuse staining typical of soluble proteins (panel a and the cell shown in the right of panel g). These results suggest that anti-Ras-induced apoptosis is strictly related to the antibody-mediated aggregation of p21Ras.

We next evaluated the effect of aggregating anti-Ras $\mathrm{scFv}$ fragments on cell growth using the colony formation assay. Table 2 shows the percentage of G418- resistant colonies obtained by transfecting the established human tumor pancreatic carcinoma MIA $\mathrm{PaCa} 2$ and the breast adenocarcinoma MDA-MB-231 cells with the anti-(Ras 1) and anti-(Ras 5) scFv fragments. The percentage was calculated with respect to the number of G418-resistant colonies obtained by transfecting the control anti-( $\beta \mathrm{Gal}) \mathrm{scFv}$, indicated as $100 \%$ in Table 2. As shown, the anti-Ras scFvs induced growth suppression on both cell lines with a similar decrease in colony formation efficiency: $34-37 \%$ for anti-(Ras 1) and 23$29 \%$ for anti-(Ras 5).

\section{Aggregating anti-Ras scFvs enhance aggresome formation and proteasome recruitment}

Overexpression of $\mathrm{scFv}$ fragments in the cytoplasm leads to the formation of large perinuclear aggresomes rich in ubiquitinated-scFv fragments [18]. Aggresome formation is thought to be a general response that occurs in the cell whenever the degradative capacity of the proteasome is exceeded. It is thought to be a symptom of saturation of the UPS [12,13]. To verify whether the expression of aggregating anti-Ras induces proteasome impairment and enhances aggresome formation, we transfected NIH 3 T3 Ki-Ras with anti-(Ras 1), anti-(Ras 5) and two irrelevant scFvs: the soluble anti- $\beta$ Gal and the strongly aggregating anti-NGF $\mathrm{scFv}$ fragment. The histogram in Fig. $2 \mathrm{~A}$ shows striking up-regulation of aggresome formation induced by anti-Ras scFvs; values of $57-62 \%$ for anti(Ras 5) and 65-75\% for anti-(Ras 1) were reached $48 \mathrm{~h}$ after transfection. In contrast, no aggresomes were present in cells transfected with the soluble anti- $\beta$ Gal scFv, and 10-13\% was observed in anti-NGF-transfected cells, a value comparable to that reported for other aggregating peptides [22]. In the latter case, only inhibition of proteasome activity with $1 \mu \mathrm{M}$ lactacystin induced aggresome formation in $60-65 \%$ of transfected cells (data not shown).

In addition to a major aggregated protein species, aggresomes are enriched in molecular chaperones, chaperonins and proteasomes subunits. Thus, proteasome recruitment has been described as a fundamental step of aggresome biogenesis [12,18,23-25]. To compare the process of proteasome recruitment induced by aggregating scFvs, we studied the intracellular distribution of the $20 \mathrm{~S}$ core proteasome in transfected cells. Figure 2B shows double immunofluorescence of NIH 3T3 Ki-Ras cells transfected with anti-NGF (a, b and c), anti-(Ras 5) (d, e and $\mathrm{f}$ ) and anti-(Ras 1) (g, h and i). The scFv fragments were immunolabeled with the anti-myc IgG $9 \mathrm{E} 10$ (a, d and g) and the antibody to $20 \mathrm{~S}$ proteasome core components (b, e and h). Panels $\mathrm{c}, \mathrm{f}$ and $\mathrm{i}$ illustrate the combination of the two chromophores in a single image in which the sites of colocalization are shown in yellow. It can be seen that anti-Ras scFvs tended to form aggregates which colocalized with the 20S proteasome subunits (panels $\mathrm{f}$ and i). In particular, a markedly altered distribution of $20 \mathrm{~S}$ proteasome core was evident in both cases. In contrast, although anti-NGF scFvs formed many aggregates (panel a), the 20S proteasome core was not recruited into these structures (panel c), suggesting that aggregating anti-Ras scFvs specifically led 
A
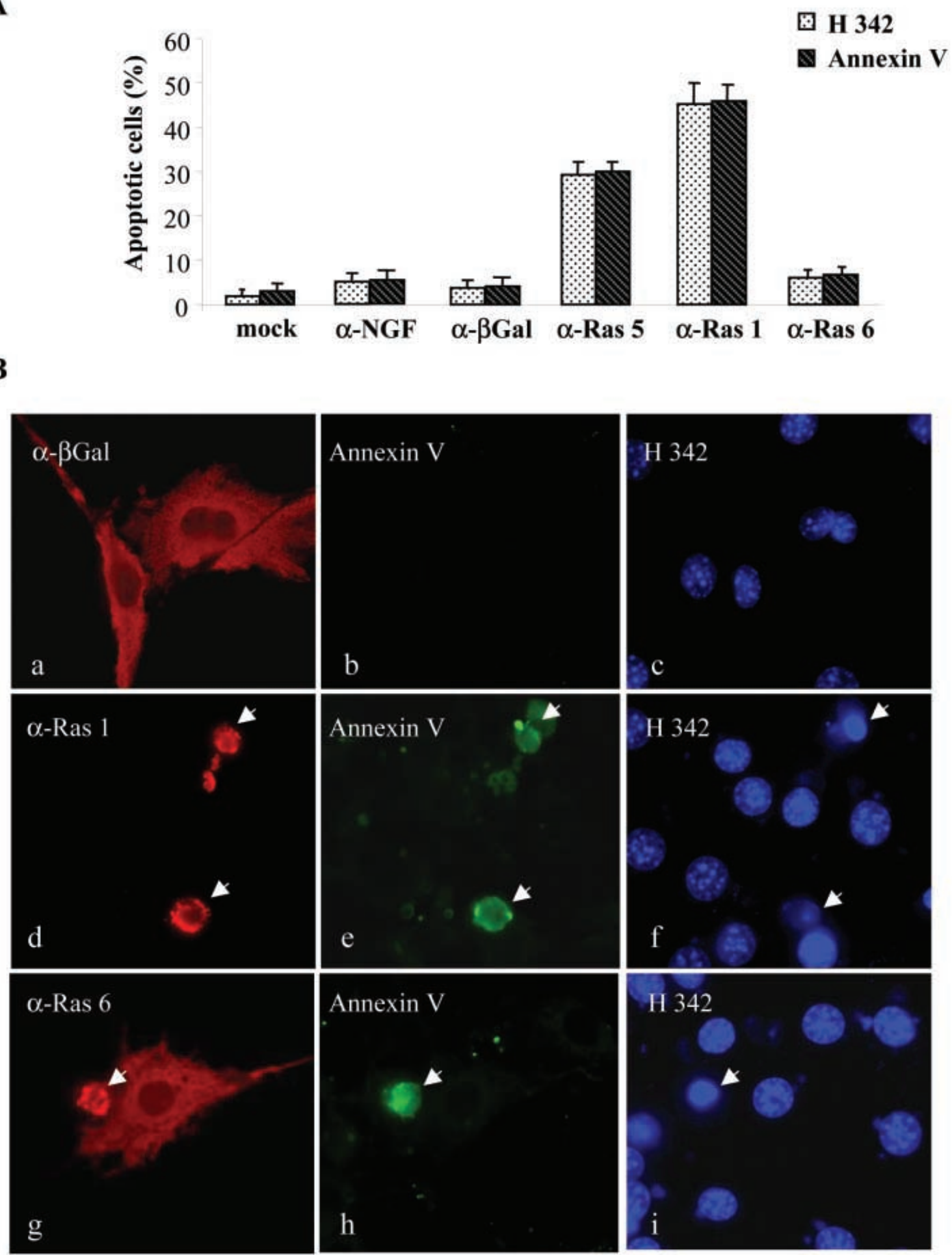

Fig. 1. Aggregating anti-Ras scFvs fragments induce apoptosis. (A) Annexin V/Hoechst 33342-positive cells were counted in mock-transfected cells and cells transfected with different $\mathrm{scFv}$ fragments (as indicated), $48 \mathrm{~h}$ after transfection. The histogram shows the mean of three different experiments in which at least 100-150 positively transfected cells were counted. (B) Three representative fields of NIH 3T3 Ki-Ras cells transfected with the irrelevant anti-( $(\mathrm{Gal})$ (a, b and c), anti-(Ras 1$)(\mathrm{d}, \mathrm{e}$ and $\mathrm{f})$ and anti-Ras $6 \mathrm{scFv}(\mathrm{g}, \mathrm{h}$ and i) fragments. Cells were first costained in vivo with annexin V (b, e and h) and Hoechst 33342 dye (c, f and i) and, successively, viewed by immunofluorescence with anti-myc IgG (mab 9E10) (a, d and g) to reveal scFv-positive cells. Arrows indicate apoptotic cells. 
Table 1. Effect of anti-Ras scFv fragments on cell survival. The percentage of apoptotic cells detected are shown for three cell lines, transfected with different $\mathrm{scFv}$ fragments using annexin $\mathrm{V}$ and Hoechst 33342 combined staining. The results represent the mean from three different experiments in which at least $100-150$ positively transfected cells were counted. nt, Not transfected; nd, not determined.

\begin{tabular}{lccl}
\hline & 3T3 K-Ras & Ger & MIA PaCa-2 \\
\hline nt & $2 \pm 1$ & $3 \pm 1$ & $4 \pm 2$ \\
$\alpha$ - $\beta$ Gal & $3 \pm 0.5$ & nd & nd \\
$\alpha$-NGF & $4 \pm 1$ & $12 \pm 0.5$ & $9 \pm 0.6$ \\
$\alpha$-Ras 1 & $45.8 \pm 5$ & $20 \pm 3$ & nd \\
$\alpha$-Ras 5 & $30 \pm 3.5$ & $49.5 \pm 2$ & $43.6 \pm 3.5$ \\
\hline
\end{tabular}

Table 2. Colony formation assay. The number of G418-resistant colonies of two human tumor cell lines transfected with different $\mathrm{scFv}$ fragments is shown. The percentage is measured with respect to the number of colonies formed in anti- $\beta$ Gal-transfected cells, indicated as $100 \%$. The results represent the mean of two independent experiments.

\begin{tabular}{lll}
\hline & MIA PaCa-2 & MDA-MB-231 \\
\hline$\alpha$ - $\beta$ Gal & $1536 \pm 100$ & $315 \pm 32$ \\
& $(100 \%)$ & $(100 \%)$ \\
$\alpha$-Ras 1 & $1014 \pm 120$ & $198 \pm 18$ \\
& $(66 \%)$ & $(63 \%)$ \\
$\alpha$-Ras 5 & $1184 \pm 81$ & $224 \pm 24$ \\
& $(77 \%)$ & $(71 \%)$ \\
\hline
\end{tabular}

to alteration of proteasome distribution and enhancement of proteasome recruitment to the newly formed aggregates.

\section{Anti-Ras scFv fragments inhibit proteasome activity}

The up-regulation of aggresome formation and the altered proteasome distribution prompted us to investigate the impact of anti-Ras scFv expression on proteasome function. To measure the proteasome activity in transfected cells, we developed an experimental protocol based on the degradation of an ectopically expressed protein specifically degraded by the UPS.

We transfected NIH 3T3 Ki-Ras fibroblasts with the single-chain $\alpha \mathrm{D} 11-\mathrm{sec}$, a soluble protein expressed in the secretory compartment [26]. Soluble and insoluble fractions of these cells treated or not with the highly specific proteasome inhibitor lactacystin [27] were analysed by Western blotting. As can be seen in Fig. 3A, accumulation of the $32-\mathrm{kDa}$ protein, corresponding to the single-chain $\alpha \mathrm{D} 11-\mathrm{sec}$, was induced by treatment with lactacystin (lane 3 ), and a ladder of higher-molecular-mass bands was clearly visible when the same blot was probed with anti-ubiquitin (see asterisks in lanes 3 and 6). Moreover, analysis of the insoluble pool confirmed the accumulation of the singlechain $\alpha \mathrm{D} 11-\mathrm{sec}$ as a ladder of higher-electrophoreticmobility bands (lane 9). Together these results indicate that the single-chain $\alpha \mathrm{D} 11-\mathrm{sec}$ was ubiquitinated and its degradation specifically inhibited by lactacystin. Therefore this molecule represents a suitable reporter of proteasome activity, which we have named single-chain proteasome substrate (scPs).

To determine whether expression of the aggregating antiRas $\mathrm{scFv}$ fragments inhibits proteasome function in vivo, we first cotransfected NIH $3 \mathrm{~T} 3 \mathrm{Ki}$-Ras with $5 \mu \mathrm{g}$ DNA plasmid encoding for scPs reporter and different amounts of GFP-tagged scFv fragments, as indicated in Fig. $3 \mathrm{~B}$ in a plasmid titration experiment. A nonaggregating antibody was used as negative control. scPs indeed accumulated only in cells transfected with anti-(Ras 1-GFP) (lanes 4 and 5). It is worth noting that transfection of cells with the scPs alone (lane 1) and cotransfection of up to $5 \mu \mathrm{g}$ of the irrelevant anti-( $\beta$ Gal-GFP) construct (lanes 2 and 3 ) did not influence the intracellular level of the scPs.

To quantify the inhibition of proteasome activity caused by the aggregating $\mathrm{scFv}$ fragments, we compared the scPs band $(32 \mathrm{kDa})$ in anti-Ras-transfected cells with the band accumulated by lactacystin treatment of anti( $\beta$ Gal-GFP)-expressing cells in the same experiment. NIH 3T3 Ki-ras fibroblasts were cotransfected with $5 \mu \mathrm{g}$ DNA encoding scPs and $5 \mu \mathrm{g}$ of the irrelevant anti$(\beta \mathrm{Gal}-\mathrm{GFP}) \mathrm{scFv}$, incubated or not with different concentration of lactacystin for $6 \mathrm{~h}$ to induce a doseresponse accumulation of scPs (Fig. 3C, lanes 4, 5 and 6). The scPs band started to accumulate at $1 \mu \mathrm{M}$ lactacystin (lane 5) and its intensity increased 3-4 times, on average, at $5 \mu \mathrm{M}$ (lane 6).

As can be seen, anti-(Ras 1) induced stronger inhibition of proteasome activity than that obtained with $5 \mu \mathrm{M}$ lactacystin (compare the scPs band in lanes 3 and 6 of Fig. 3C), and anti-(Ras 5) induced stronger inhibition of proteasome activity than that obtained with $1 \mu \mathrm{M}$ lactacystin (compare lanes 2 and 5). Note that an aggregating molecule, such as the irrelevant anti-NGF scFv fragment, induced detectable accumulation of scPs, comparable to that obtained with $1 \mu \mathrm{M}$ lactacystin (compare lanes 1 and 5). Equal amounts of protein were loaded in the gel, as revealed by Coomassie blue staining (not shown) and by anti-HP1 $\beta$ immunoblotting (Fig. 3B,C). Transfection efficiency of scFv-GFP constructs was controlled by anti-GFP immunoblotting (Fig. 3C).

\section{Lactacystin in combination with anti-Ras scFvs synergistically induces apoptosis}

So far we have shown that aggregating anti-Ras scFv fragments induce apoptosis, and this phenomenon appears to be related to proteasome dysfunction. As it has also been recently demonstrated that proteasome inhibitors induce apoptosis in a variety of human tumor types [16,17], we decided to investigate the effect of anti-Ras scFvs and lactacystin, in combination, on cell survival.

We therefore transfected NIH 3T3 Ki-Ras cells with different scFvs fragments and analysed the percentage of annexin $\mathrm{V} /$ Hoechst-positive cells in the absence or presence of subtoxic doses of lactacystin for $24 \mathrm{~h}$, as specified (Fig. 4). As shown, $1 \mu \mathrm{M}$ lactacystin concentration did not induce apoptosis per se in mock-transfected cells and in cells transfected with two irrelevant $\mathrm{scFv}$ fragments, the mostly soluble anti-( $\beta \mathrm{Gal})$ and the strongly aggregating anti-NGF. Strikingly, when this concentration of lactacystin was added to anti-(Ras 5)-transfected cells, it produced a marked 
A

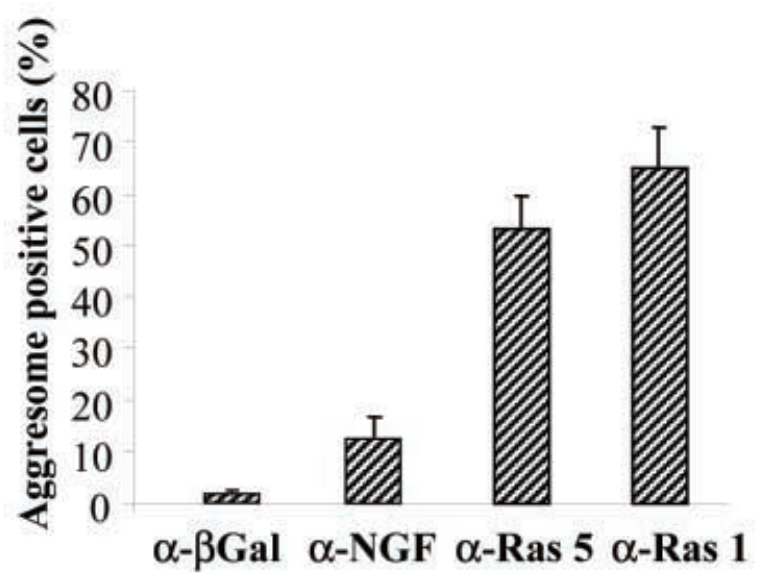

B
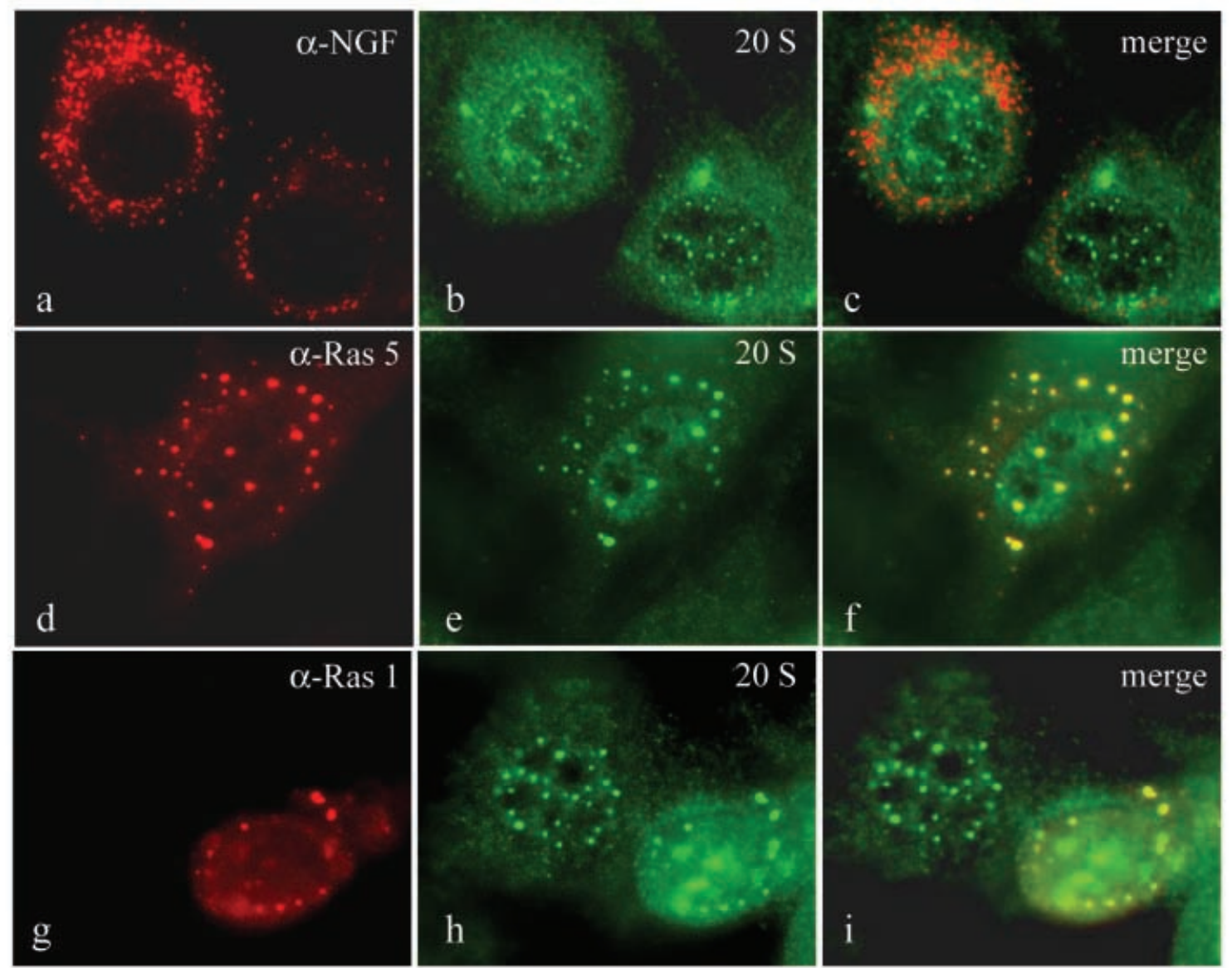

Fig. 2. Aggresome formation and proteasome recruitment induced by anti-Ras scFvs. (A) NIH 3T3 Ki-Ras cells were transfected with different scFv fragments (as indicated). Aggresome formation was followed by indirect immunofluorescence with anti-myc IgG (mAb 9E10). The histogram shows the percentage of aggresome-positive cells $48 \mathrm{~h}$ after transfection. At least 200 positively transfected cells were counted for each experiment. (B) NIH 3T3 Ki-Ras cells transfected with anti-NGF (a, b and c), anti-(Ras 5) (d, e and f) and anti-(Ras 1) (g, h and i) were double immunolabeled with mouse anti-myc IgG, 9E10 (a, d and g) and rabbit anti-(20S proteasome core) (b, e and h). Panels c, f and i represent the merged images.

cytotoxic response. Thus, $70-75 \%$ of cells became apoptotic, which is higher than the sum of effects due to either agent alone, indicating synergistic cytotoxicity of the combined treatment.

\section{Effect of dominant-negative N17-H-ras on apoptosis}

To investigate whether suppression of p21Ras function, per se, by a dominant-negative molecule is cytoxic, we used 
A

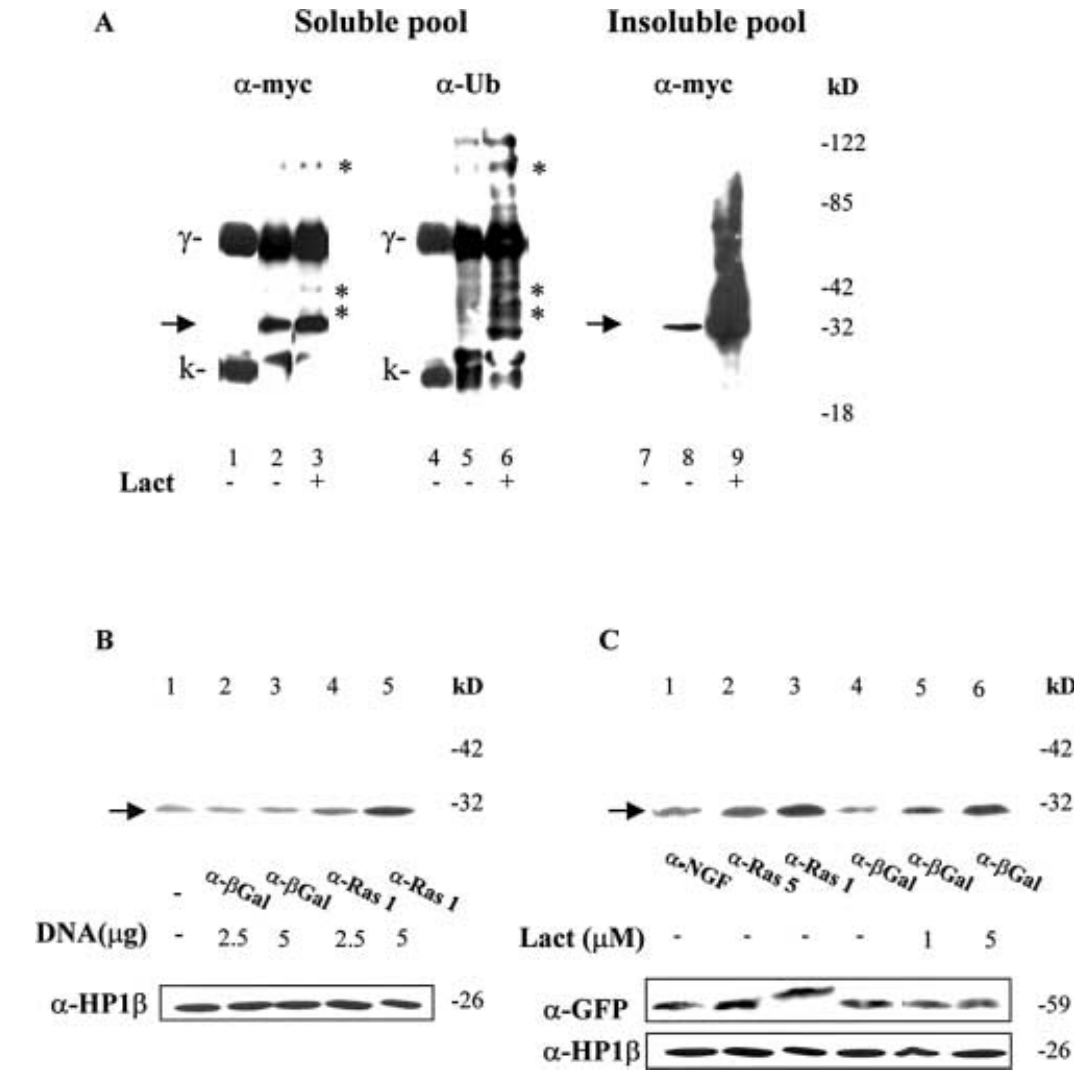

Soluble pool

Insoluble pool

Fig. 3. Proteasome activity assay in transfected cells. (A) Western blot analysis of soluble and insoluble pool extracts of NIH $3 \mathrm{~T} 3 \mathrm{Ki}-\mathrm{Ras}$ cells transfected with the scPs and incubated or not with $5 \mu \mathrm{M}$ lactacystin, as specified. The soluble pool was first anti-myc-immunopurified and then viewed with anti-myc $\operatorname{IgG}$ (lanes 1, 2 and 3) and anti-ubiquitin $\operatorname{IgG}$ (lanes 4, 5 and 6). The insoluble pool was revealed with anti-myc IgG (lanes 7, 8 and 9). The migration of molecular mass markers (in kDa) and of the heavy ( $\gamma$ ) and light (k) IgG chains are indicated. Arrows point to the scPs, and the asterisks denote three ubiquitinated bands of the scPs. Overloading the gel up to three times allowed us to highlight the scPs-ubiquitinated bands in lanes 6 and 9. (B) Western blot analysis of total cell extract of NIH 3T3 Ki-Ras cells cotransfected with the myc-tagged scPs without (lane 1) or with different amounts of anti- $\beta$ Gal (lanes 2 and 3) or anti-(Ras 1) (lanes 4 and 5) scFv-GFP DNA plasmids, as indicated. Blots were detected using anti-myc IgG (9E10) and anti-HP1 $\beta$ IgG. The arrow indicates the scPs protein. (C) Western blot analysis of total cell extract of NIH 3T3 Ki-Ras cells cotransfected with the myc-tagged scPs and the anti-NGF (lane 1), anti-(Ras 5) (lane 2), anti-(Ras 1) (lane 3) or irrelevant anti-( $\beta$ Gal-GFP) $\mathrm{scFv}$ (lane 4), incubated with 1 or $5 \mu \mathrm{m}$ lactacystin for $6 \mathrm{~h}$ (lanes 5 and 6 , respectively). Blots were detected using anti-myc IgG, anti-GFP IgG and anti-HP1 $\beta \mathrm{IgG}$. The arrow indicates the proteasome substrate band. Equal amounts of protein were loaded for each experimental point. GFP-fused $\mathrm{scFv}$ expression was monitored by anti-GFP IgG, and protein level by anti-HP1 $\beta$ IgG.

the N17-H-ras mutant. This molecule has been shown to efficiently block Ras function in vivo, because of its preferential affinity for GDP [19].

We transfected NIH 3T3 Ki-Ras cells with N17-H-ras and different $\mathrm{scFv}$ fragments and counted the annexin $\mathrm{V}$ (Alexa 568)/Hoechst-positive cells. In this experiment, all constructs were tagged with GFP and under the same promoter (cytomegalovirus). The $\mathrm{scFv}$ moiety in GFPtagged scFvs has been shown to be functional in vivo [18], and the GFP-N17-H-ras mutant maintained its neutralizing activity (data not shown). As shown in Fig. 5, the percentage of apoptosis in cells expressing the dominant-negative N17-H-ras was 25-30, a value only slightly higher than that observed by transfection with the irrelevant anti-( $\beta \mathrm{Gal}-$ GFP) $\mathrm{scFv}(18-20 \%)$. In contrast, expression of the anti-(Ras 5)-GFP scFv fragment led to a much greater apoptotic effect $(65 \%)$. It is worth noting that transfection of cells with the dominant-negative N17-H-ras alone did not induce aggregates or aggresomes, and its cotransfection with the strongly aggregating scFvs did not influence their capacity to form aggresomes (unpublished observation).

This finding indicates that displacing the endogenous p21Ras by a dominant-negative mutant per se is not as cytotoxic as diverting it to $\mathrm{scFv}$ aggresomes.

\section{Discussion}

In this paper we show that expression of highly aggregating anti-p21Ras scFv fragments causes apoptosis in a large percentage of transfected cells. This cytotoxic effect is strictly related to the aggregation state of the $\mathrm{scFv}$ fragments, irrespective of the binding affinity and p21Ras epitope recognized by the $\mathrm{scFv}$. Thus, the binding affinity of the aggregating $\mathrm{scFv}$ fragments presented in this study varies between $4 \mathrm{~nm}$ and $2 \mu \mathrm{M}$ [anti-(Ras 1) and anti(Ras 5), respectively] [9], and appears therefore not to be a 


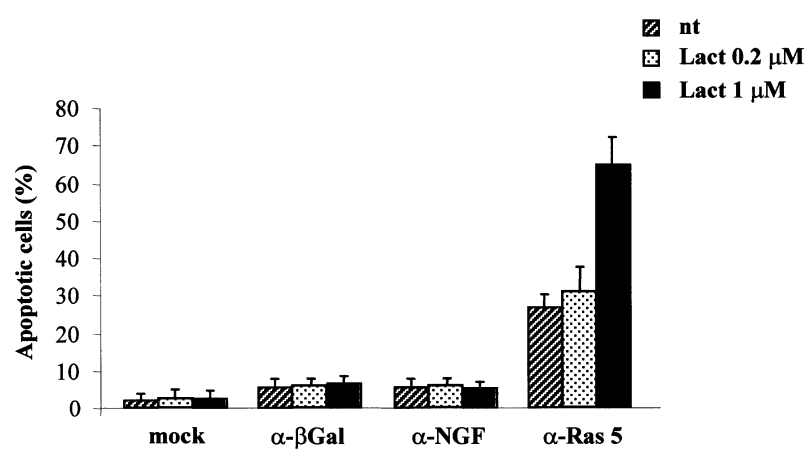

Fig. 4. Combined anti-Ras scFvs/lactacystin treatment synergistically induces apoptosis. Annexin V/Hoechst 33342-positive cells were counted in mock-transfected cells and cells transfected with $\mathrm{scFv}$ fragments (as indicated), treated or not with 0.2 or $1 \mu \mathrm{M}$ lactacystin for $24 \mathrm{~h}$. The histogram shows the mean of three different experiments in which at least $100-150$ positively transfected cells were counted.

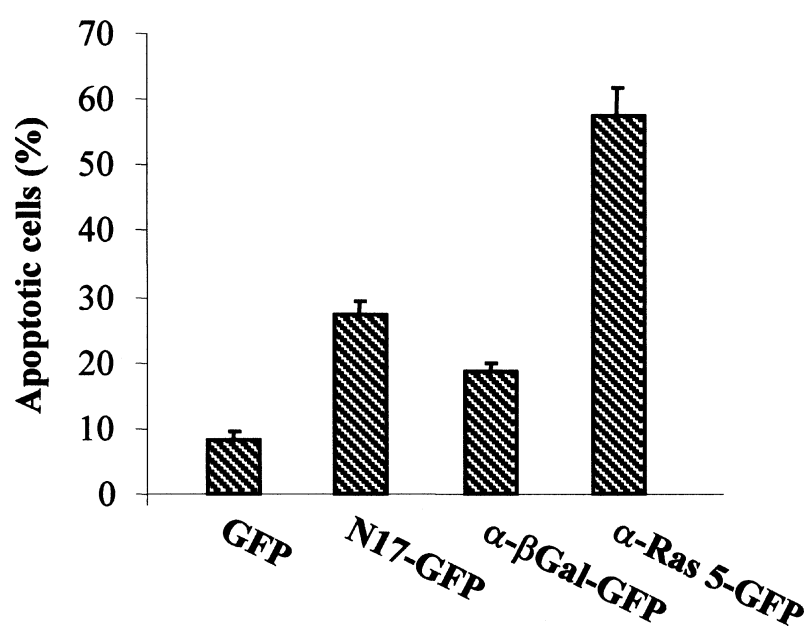

Fig. 5. Effect of dominant-negative N17-H-ras and anti-Ras scFv fragments on cell death. Apoptotic cells (detected using annexin V/ Hoechst combined staining) were counted in cells transfected with the dominant-negative N17-H-ras, anti-( $(\mathrm{Gal})$ and anti-(Ras 5) $\mathrm{scFv}$ fragments (as indicated), all tagged with GFP, and with GFP alone as a control. Means from three different experiments are shown. At least $100-150$ positively transfected cells were counted.

critical parameter for achieving intracellular inhibition of Ras function in vivo and/or induction of apoptosis. Moreover, unlike anti-(Ras 1), anti-(Ras 5) does not neutralize Ras function in vitro. Thus, this $\mathrm{scFv}$ is not able to interfere with the intrinsic GTPase activity of p21Ras nor prevent its interaction with the Raf effector [9].

Two parallel cellular and biochemical processes appear to be responsible for the anti-Ras-mediated apoptosis described in this paper: (a) impairment of the UPS by $\mathrm{scFv}$ aggregation; (b) antibody-mediated targeting of p21Ras to the UPS.

It is well known that cytosolic scFvs fragments have different solubility and stability properties, which crucially depend on their primary sequence. Notwithstanding their propensity to form aggregates, these molecules are variably ubiquitinated and targeted to the UPS to be degraded [18].
We studied three scFv fragments, anti-NGF, anti-(Ras 1) and anti-(Ras 5), which are fully aggregating molecules both in vitro and in vivo, but show variability in terms of cytotoxicity.

We found that the irrelevant, strongly aggregating anti$\mathrm{NGF} \mathrm{scFv}$ fragment induces apoptosis, which varies from $5 \%$ to $8 \%$ of transfected cells in different cell lines (Fig. 1 and Table 1). Interestingly, most of the apoptotic cells show clearly defined immunoreactive $\mathrm{scFv}$ aggresomes, shrink, and exhibit condensed chromatin and phosphatidylserine translocation. Moreover, the process is accompanied by cytochrome $c$ release and activation of caspase 3 (data not shown). This appears to be a general mechanism, which is not dependent on the presence of the intracellular antigen or formation of the antigen-antibody complex.

A much more severe phenotype is observed in the case of aggregating anti-Ras scFvs, related to sequestration of the endogenous p21Ras. Thus, up to $50 \%$ of cells expressing anti-(Ras 1) undergo apoptosis (Fig. 1 and Table 1). This process is accompanied by aggresome formation in the vast majority of transfected cells and marked inhibition of proteasome function. In the case of anti-(Ras 1), for example, over $70 \%$ of cells show aggresomes after $48 \mathrm{~h}$ of transfection (Fig. 2), and up to $100 \%$ show aggresomes after $72 \mathrm{~h}$ of transfection (data not shown). Moreover, in this cell population, proteasome activity is strongly inhibited, as measured by intracellular accumulation of the proteasome substrate scPs (Fig. 3). The marked difference in cell lethality between anti-NGF and anti-Ras scFv expression may be attributed not only to the direct neutralization of Ras function, but also to the diversion of p21Ras to the UPS $[9,18]$ which is associated with antibodymediated coaggregation of Ras-binding partners.

It is worth mentioning that the soluble anti-(Ras 6) $\mathrm{scFv}$ fragment inhibits cell proliferation without inducing apoptosis. Interestingly, only cells that exhibit $\mathrm{scFv}$ aggregates show an apoptotic phenotype (Fig. 1B). Moreover, the dominant-negative N17-H-ras, which is mostly soluble and does not induce aggresome formation and proteasome impairment, only slighly affects cell viability. So, the aggregation state of the anti-Ras scFvs seems to be the crucial event to the induction of cell death.

In line with our findings, several reports have suggested that the expression of pathological aggregating-prone molecules, such as cystic fibrosis transmembrane conductance regulator, huntingtin, parkin and prion $[22,24,25,28]$, results in aggresome formation and impairment of the UPS and, in some cases, apoptosis [24,28]. For example, expression of polyglutamine-expanded huntingtin fragment leads to redistribution of proteasomes from the total cellular environment to the huntingtin aggregates and to a higher rate of aggresome formation. Consequently, there is a decrease in proteasome availability for degrading other key target proteins. Furthermore, the altered proteasomal function is associated with apoptosis through disruption of mitochondrial membrane potential and cytochrome $c$ release [24].

The causal relation between scFv aggregation, p21Ras sequestration, proteasome dysfunction and cytotoxicity is further demonstrated by the combined treatment with antiRas scFvs and lactacystin. This potent drug belongs to the class of proteasome inhibitors, which inhibit the 
degradation of multi-ubiquitinated target proteins (i.e. cell cycle regulatory proteins, such as cyclins and cyclindependent kinase inhibitor) and induces apoptosis in different tumor cells. These molecules represent potential new anticancer agents [16,17]. Strikingly, we found that a nontoxic dose of lactacystin, only one-tenth of its $\mathrm{IC}_{50}$, induces a synergistic apoptotic effect in anti-Ras-expressing cells (Fig. 4). This result underlines proteasome dysfunction as the specific event in anti-Ras-induced apoptosis. A combinatorial approach, consisting of a cancer-specific apoptosis-inducing gene and proteasome inactivation, may be a good rationale for evaluating new strategies for cancer therapy.

\section{Acknowledgements}

We are grateful to R. Orlandi and R. Testi for helpful suggestions, and $\mathrm{S}$. Nasi for providing the N17-H-ras mutant. Funding for this work was through a European Commission grant (QLG2-CT-2000-00345). A.C. and I.F. acknowledge fellowships from the same EC grant.

\section{References}

1. Campbell, S.L., Khosravi-Far, R., Rossman, K.L., Clark, G.J. \& Der, C.J. (1998) Increasing complexity of Ras signaling. Oncogene 17, 1395-1413.

2. Adjei, A.A. (2001) Blocking oncogenic Ras signaling for cancer therapy. J. Natl. Cancer Inst. 93, 1062-1074.

3. Canevari, S., Biocca, S. \& Figini, M. (2002) Re: Blocking oncogenic Ras signaling for cancer therapy. J. Nat. Cancer Inst. 94, 1031-1032.

4. Cattaneo, A. \& Biocca, S. (1997) Intracellular Antibodies: Development and Applications, pp. 1-196. Springer Verlag, Berlin.

5. Richardson, J.H. \& Marasco, W.A. (1995) Intracellular antibodies: development and therapeutic potential. Trends Biotechnol. 13, 306-310.

6. Cardinale, A., Lener, M., Messina, S., Cattaneo, A. \& Biocca, S. (1998) The mode of action of Y13-259 scFv fragment intracellularly expressed in mammalian cells. FEBS Lett. 439, 197-202.

7. Cochet, O., Kenigsberg, M., Delumeau, I., Virone-Oddos, A., Multon, M.C., Fridman, W.H., Schweighoffer, F., Teillaud, J.L. \& Tocqué, B. (1998) Intracellular expression of an antibody fragment-neutralizing p21 Ras promotes tumor regression. Cancer Res. 58, 1170-1176.

8. Cattaneo, A. \& Biocca, S. (1999) The selection of intracellular antibodies. Trends Biotechnol. 17, 115-121.

9. Lener, M., Horn, I.R., Cardinale, A., Messina, S., Nielsen, U.B., Rybak, S.M., Hoogenboom, H.R., Cattaneo, A. \& Biocca, S. (2000) Diverting a protein from its cellular location by intracellular antibodies. The case of p21Ras. Eur. J. Biochem. 267, 1196-1205.

10. Filesi, I., Cardinale, A., Van der Sar, S., Cowell, I.G., Singh, P. \& Biocca, S. (2002) Loss of Heterochromatin Protein 1 (HP1) chromodomain function in mammalian cells by intracellular antibodies causes cell death. J. Cell Sci. 115, 1803-1813.

11. Yi, K.S., Chung, J.H., Lee, Y.H., Chung, H.G., Kim, I.J., Suh, B.C., Kim, E., Cocco, L., Ryu, S.H. \& Suh, P. (2001) Inhibition of the EGF-induced activation of phospholipase $\mathrm{C}-\gamma 1$ by a single chain antibody fragment. Oncogene 20, 7954-7964.

12. Johnston, J.A., Ward, C.L. \& Kopito, R.R. (1998) Aggresomes: a cellular response to misfolded proteins. J. Cell Biol. 143, 18831898.

13. Kopito, R.R. \& Sitia, R. (2000) Aggresomes and Russel bodies. Symptoms of cellular indigestion? EMBO Rep. 1, 225-231.

14. Ciechanover, A., Orian, A. \& Schwartz, L.A. (2000) Ubiquitinmediated proteolysis: biological regulation via destruction. Bioessay 22, 442-451.

15. Voges, D., Zwickl, P. \& Baumeister, W. (1999) The 26S proteasome: a molecular machine designed for controlled proteolysis. Annu. Rev. Biochem. 68, 1015-1068.

16. Adams, J., Palombella, V.J., Sausville, E.A., Johnson, J., Destree, A., Lazarus, D.D., Maas, J., Pien, C.S., Prakash, S. \& Elliott, P.J. (1999) Proteasome inhibitors: a novel class of potent and effective antitumor agents. Cancer Res. 59, 2615-2622.

17. Goldberg, A.L. \& Rock, K. (2002) Not just research tools: proteasome inhibitors offer therapeutic promise. Nat. Med. 8, 338-340.

18. Cardinale, A., Filesi, I. \& Biocca, S. (2001) Aggresome formation by anti-Ras intracellular $\mathrm{scFv}$ fragments: the fate of the antigenantibody complex. Eur. J. Biochem. 268, 268-277.

19. Feig, L. \& Cooper, G.M. (1988) Inhibition of NIH 3 T3 cell proliferation by a mutant ras protein with preferential affinity for GDP. Mol. Cell Biol. 8, 3235-3243.

20. Biocca, S., Ruberti, F., Tafani, M., Pierandrei-Amaldi, P. \& Cattaneo, A. (1995) Redox state of single-chain Fv fragments targeted to endoplasmic reticulum, cytosol and mitochondria. Bio/Technology 13, 1110-1115.

21. Wreggett, K.A., Hill, F., James, P.S., Hutchings, A., Butcher, G.W. \& Singh, P.B. (1994) A mammalian homologue of Drosophila heterochromatin protein 1 (HP1) is a component of constitutive heterochromatin. Cytogenet. Cell Genet. 66, 99-103.

22. Bence, N.F., Sampat, R.M. \& Kopito, R.R. (2001) Impairment of the ubiquitin-proteasome system by protein aggregation. Science 292, 1552-1555.

23. Garcia-Mata, R., Bebok, Z., Sorscher, E.J. \& Sztul, E.S. (1999) Characterization and dynamics of aggresome formation by a cytosolic GFP-chimera. J. Cell Biol. 146, 1239-1254.

24. Jana, N.R., Zemskov, E.A., Wang, G. \& Nukina, N. (2001) Altered proteasomal function due to the expression of polyglutamine-expanded truncated $\mathrm{N}$-terminal huntingtin induces apoptosis by caspase activation through mitochondrial cytochrome C release. Hum. Mol. Genet. 10, 1049-1059.

25. Junn, E., Lee, S.S., Suhr, U.T. \& Mouradian, M.M. (2002) Parkin accumulation in aggresomes due to proteasome impairment. J. Biol. Chem. 277, 47870-47877.

26. Ruberti, F., Bradbury, A. \& Cattaneo, A. (1993) Cloning and expression of an anti-NGF antibody for studies using the neuroantibody approach. Cell Mol. Neurobiol. 13, 559-568.

27. Fenteany, G. \& Schreiber, S.L. (1998) Lactacystin, proteasome function and cell fate. J. Biol. Chem. 273, 8545-8548.

28. Ma, J., Wollmann, R. \& Lindquist, S. (2002) Neurotoxicity and neurodegeneration when PrP accumulates in the cytosol. Science 298, 1781-1785. 